University of Wollongong

Research Online

Faculty of Engineering and Information

Faculty of Engineering and Information

Sciences - Papers: Part A

Sciences

$1-1-2016$

Learning network storage curriculum with experimental case based on embedded systems

Qingguo Zhou

Lanzhou University, zhouqg@lzu.edu.cn

Jiong Wu

Lanzhou University

Ting Wu

Lanzhou University

Jun Shen

University of Wollongong, jshen@uow.edu.au

Rui Zhou

Lanzhou University

Follow this and additional works at: https://ro.uow.edu.au/eispapers

Part of the Engineering Commons, and the Science and Technology Studies Commons

Research Online is the open access institutional repository for the University of Wollongong. For further information contact the UOW Library: research-pubs@uow.edu.au 


\title{
Learning network storage curriculum with experimental case based on embedded systems
}

\begin{abstract}
In this paper, we present an experimental case for the course of "Network Storage and Security," which benefited from an improved learning outcome for our students. The newly designed experiments-based contents are merged into the current course to help students obtain practical experiences about network storage. The experiments aim to build a network storage system based on available resources instead of any specialized network storage equipment. Technically, students can learn general practical knowledge of network storage on iSCSI (a network storage protocol based on IP technology) and also the technologies of embedded system. Through the experimental case, we found that it could fully enhance students' comprehensive and practical abilities, develop students' teamwork spirit and creativity, and especially improve the learning outcome of network storage curriculum. These learning and thinking methods can also be generalized and applied to other computer science related courses.
\end{abstract}

\section{Keywords}

embedded, case, experimental, systems, curriculum, learning, storage, network

Disciplines

Engineering | Science and Technology Studies

\section{Publication Details}

Zhou, Q., Wu, J., Wu, T., Shen, J. \& Zhou, R. (2016). Learning network storage curriculum with experimental case based on embedded systems. Computer Applications in Engineering Education, 24 (2), 186-194. 


\title{
Learning Network Storage Curriculum with Experimental Case based on Embedded Systems
}

\author{
Qingguo ZHOU, Jiong Wu, Ting Wu, Jun Shen, Rui ZHOU ${ }^{1}$
}

\begin{abstract}
In this paper, we present an experimental case for the course of 'Network Storage and Security', which benefited from an improved learning outcome for our students. The newly designed experiments based contents are merged into the current course to help students obtain practical experiences about network storage. The experiments aim to build a network storage system based on available resources instead of any specialized network storage equipment. Technically, students can learn general practical knowledge of network storage on iSCSI (a network storage protocol based on IP technology) and also the technologies of embedded system. Through the experimental case, we found that it could fully enhance students' comprehensive and practical abilities, develop students' teamwork spirit and creativity, and especially improve the learning outcome of network storage curriculum. These learning and thinking methods can also be generalized and applied to other computer science related courses.
\end{abstract}

Keywords: Network storage; practice ability; experimental case; teaching effect;

\section{INTRODUCTION}

With the rapid development of information technologies, the computer science curricula should keep updating to offer newly developed technologies or knowledge to students [1]. In the computer science curricula, there are fundamental courses such as discrete mathematics, operating systems, data structure, digital logic, computer architecture etc. They are indispensable for students to develop the overarching concepts of computer science. Meanwhile, there are some other courses more specific in explaining one application field of computer science. These courses aim to deliver more recent developments and knowledge of computer science, or discuss some new technology trends [2-6], such as the new terminologies of Cloud computing [7, 32], mobile Internet [8], Internet of Things, etc. Though the novel knowledge is practical, the related courses are usually set up lately and mainly teaching knowledge by the rather theoretical lectures. Therefore, it is extremely difficult for students to gain practical experiences and the outcome of the teaching could not be achieved as expected.

For these courses, the obstacles for providing experimental cases may include 1) the specialized experimental equipment and the environments are not always readily available, due to the high cost; 2) the contents are being updated more frequently if compared to the fundamental courses; 3) teachers themselves are lacking practical experiences; 4) complicated management procedure is required to change the curricula to adopt the new courses.

In this study, we are concerned with the explosion of information and data, where storage technology has become one of the major parts of IT infrastructure $[9,10]$. It has been evolving continuously as an important role in the wide information system and Internet deployment [11]. Meanwhile, different types of storage equipment have emerged one after another. The development in storage technology has raised new requirements for students studying in Computer Sciences. On the one hand, the traditional storage method is unable to meet the need of new data-intensive applications and networked enterprise applications. On the other hand, new network storage technology related to data-centralized management, data security, data flexibility, data availability and other aspects becomes particularly important. Now it is treading to transmit the online data to networked storage, so the IT sector needs a large number of network storage experts.

Because of the popularity of and wide attention to network storage[14-16], Lanzhou University has recently set up the curriculum of 'Network Storage and Security' for undergraduates in computer-related majors [12, 13]. The course profoundly introduces the rising network storage system to students, including hardware, network protocol and technology, operating system and professional software, in order to comprehensively develop students' knowledge on

\footnotetext{
${ }^{1}$ The first author, the second, third author and the last author are with School of Information Science \& Engineering, Lanzhou University, Lanzhou, 73000,Gansu, China(e-mail:\{zhouqg,jwu2009,wut13,zr\}@ lzu.edu.cn)

The fourth author is with 3.School of Information Systems and Technology, University of Wollongong, Wollongong, NSW, 2522, Australia (e-mail: \{ jshen\}@uow.edu.au)
} 
related network storage technologies [17]. However, the course is mainly composed of theoretical lectures throughout one single semester. According to the feedback of previous semesters, it was difficult for students to get real impressions on network storage systems because they could not accumulate practical experiences. These disappointing learning outcomes resulted in an unpleasant fact, that the course could achieve the teaching goal as expected ${ }^{2}$.

Both teachers and students involved in this course have realized the cramming system [18] is unable to satisfy the cultivation requirements for innovative minds, especially the majors such as computer sciences require students to gain more frequent practices. As knowledge is an initiative construction by learners, teachers should play the role of guider and facilitator more often so that students can not only acquire knowledge from lectures, but also actively participate in the teaching and learning activities [19]. An effective way is to build global knowledge framework by students themselves, under teachers' essential guidance. Therefore, in order to achieve the comprehensiveness in both theory and practice, it becomes critical to merge practical contents into the current course.

We consider using a practice case to enhance the teaching outcome of the network storage curriculum. In our course, teachers not only impart theory to the students, but also combine the theory with practice to guide students in building network storage system. Firstly, the using of Intel Atom processor platform decreased the difficulties of network storage system building, it make the student implement it during the class easily. Secondly, the practice case ensures the teacher guidance student in experiment face to face, the problems created in experiment will be solved immediately. Thirdly, the communication between students will improve the students' team cooperation skill, it help students better integrated into the classroom. At last, it can assist them to discover and summarize effective learning patterns consciously, and as a result, they can feel the joy of learning by exploring their own potentialities.

For the practical contents, what the course needs is small-scale content, which can achieve learning goals, instead of large-scale or professional environment of network storage, which is too difficult or complicated to access in a course. In the past, we have built a remote laboratory for embedded systems [20,21], now we integrate the developed experimental environment for network storage into this laboratory. On the other hand, it becomes a trend that small-size terminal devices, for instance, smart phones or tablets, have been connected into network. Therefore, network storage resources can also be utilized in these various forms, and we consider bringing the ideas of network storage into practice out of the traditional equipment like desktops and servers. The reason why we consider network storage from the point view of embedded system is that we need not purchase specialized equipment for the experimental environment. Actually, with the existing embedded devices, we can deliver both the knowledge of network storage and the technologies of embedded systems.

The rest of the paper is organized as: After related work the paper analyses the experiment based teaching. The following section discusses features of our case. Then the paper describes the details of the experiment case and it is divided into three parts: 1) the introduction of the system structure of the case, in order to make students understand the basic framework; 2) the experiment contents, including the iSCSI fundamental experiment and iSCSI advanced experiment; 3) the performance test of the case. The last section discusses the students' feedback of the case study after class. Finally, the paper ends with concluding remarks.

\section{RELATED WORK}

With the rapid development of science and technology, the endless improvement of the market economy and the intensive competition demand the students to quickly learn the practical abilities. The lack of practical ability makes the students feel confused on how to arrange their study schedule and take care of themselves and communicate with others. They also do not know how to combine theoretical study and hands-on practices, how to deal with unexpected events [22]. Hence the development of practical ability has a very important significance in a person's life. Zhao Yan jun et al. (2013) realized a new practice teaching system, which was established to reform the innovation practice teaching mode research of the computer fundamentals[23]. The teaching practice proved that the reformed teaching mode would improve the students' practice ability, thinking and learning ability, and stimulate the students' study initiative to a great extent, and also cultivate the students' innovation ability [24]. Watts, Huiming \& Xiaohong (2010) presented a case study of the data access control for health information system. This case study was developed to enhance Information Assurance education by providing conceptual information that was relevant and parallel to the materials, which were learned in the classroom. And the case study was also widely used in software engineering. Compared to practice ability in class, the collaborative learning is also very important, especially in a same group. 
Collaborative learning is a kind of group learning mode where members of group learn any concept or topic through communication and discussions. The LBlog [30] integrates Learning Management System (LMS) to manage the group members' profiles to evaluate the learners' learning efficiency. Their initial experiments with the LBlog indicate that it is a useful educational tool to support collaborative learning. Online collaborative communication represents a practical and constructivist method to transmit the knowledge and experience from the teacher to students, overcoming physical distance and isolation. Luis de la Torre described an extension of two open tools and provided collaborative support to virtual and remote laboratories (VRLs) [26]. There are lots of researches having combined the new technologies in special education. Many traditional assistive technologies are now transitioning from standalone devices into apps on mobile devices. As such, Hae Won Park \& Howard (2013) follows this trend by discussing transforming a tablet into an Human-Robot Interaction research platform, where their robotic system engages the user in social interaction by learning how to operate a given APP (task) using guidance from the user. As mentioned above, all the researches shared a same goal to improve the students' practice ability and enhance the class outcome.

There is a lot of research and work on network storage technology teaching. Vladan \& Timur (2008) presented content, delivery and assessment mechanisms used for an undergraduate course on Networked Storage Technology [11]. In order to overcome the complex and costly infrastructures of Physical storage area networks (SANs), Javier García \& Joaquín Entrialgo (2014) presented a lab approach for teaching SANs [31]. In China, the Department of Computer Science of Shenzhen University began to set up the 'Network Storage Technology' courses in 2006, and the teaching model had been formed in a simplistic way in 2008. According to [32], they are mainly focusing on theoretical teaching. However, the experimental part is mainly built upon the cooperation with EMC ${ }^{2}$ Corporation. They set up a "Shenzhen University - EMC ${ }^{2}$ Information Infrastructure Joint Laboratory". Based on this experimental platform, $\mathrm{EMC}^{2}$ senior technical experts also helped to complete the teaching task. It is obvious that such a laboratory requires a lot of human and material resources. In yet another similar course in Fuzhou University [33], the experimental design was just a simple removable component within the storage system to understand the internal structure, there was no corresponding network storage technology experimental platform, and hence it was also a theoretical teaching.

We herein propose the experiment case aiming to enhance learning experience of network storage curriculum and addressing the problem mentioned in the first section of this paper. We invited 30 students to attend the class, after class we asked them to fill in the questionnaire about their feedback and then we analyze the data.

\section{THE ANALYSIS OF EXPERIMENTAL TEACHING}

Student is the center of learning. Learning is not about the teachers infusing knowledge into students, but the students actively constructing the process. Teachers get involved in the student's practice as organizers, guiders and also the managers in the case, which fully mobilize the students' autonomy. Before we introduce the case itself, we analyze the main philosophy and design principles behind the experimental teaching as follows.

Learner Analysis: College students now have a strong curiosity over new things, and they are good at communicating with classmates and teachers. Our case is easily operational and practicable, and it can not only fully tap the potential of students, but also cultivate and establish student's interest in network storage and safety related courses.

Learning Situation Analysis: The students have learned the basic knowledge of network storage previously and understood some basic concepts. This course will help students to grasp and manipulate various types of storage technologies, identify and manipulate various types of devices, and develop students' teamwork ability as well. It expands the range of students' knowledge and offers new directions for student employment.

Objectives Analysis: This course is set as an experimental course after foundation network storage courses with the aim to deepen students' understanding of the basic theory. It will enhance the students' practical ability, and consolidate the learning outcome of the classes and labs. We look forward to a number of objectives to be achieved: 1) Emotional objective: we aim to exalt students' interest in the case study, and stimulate their desire for learning. 2) Knowledge objective: we help the students to understand the theoretical and practical significance of the network storage course, and to learn network storage applications and related environmental configuration. This requires students to understand and master the storage technologies that can be used to identify and manipulate various types of storage devices. 3) Skill objective: we will let students use existing resources to build a network storage case study by themselves. 


\section{THE CASE FEATURES}

The characteristics of the network storage experiment teaching case is reflected in the following aspects:

(1) Network storage technology has a strong professional prospective with extensive applications. The traditional cramming system [18] obviously cannot meet the teaching requirements; hence the design and implementation of this case are aimed to promote the change of teaching methods, from pure theory to practices related to theory. This is a platform that can push students to master the scientific approach and improve the practical ability. Teachers will feel easy to teach, while students feel easy to learn in the teaching and learning process. The learning outcome can be optimized. Our course uses the topic of combining theory with practice to bring students more relaxed and enjoyable learning experience. It is once a year.

(2) In our case, we use the Intel Atom processor platform to build the network storage system. The powerful hardware configuration and advantageous performance of the Atom experimental platform can not only ensure the functionality and performance required for each node in the network storage system to be easy to implement, but also make the scale of the whole experimental environment be effectively under control. It can be quickly constructed and easily installed in Linux operating system according to the actual experimental content and it is more conducive for students to establish an intuitive understanding of the operating system as well.

(3) Through the operation of the system configuration, students have the freedom to select the appropriate testing tools. We can build a test environment, which implement the system functionality and performance testing. In the meantime, it helps students to accumulate practical experiences of building or using network storage systems by achieving specific application services, and it enhances the students' teamwork and problem-solving abilities.

\section{THE DETAILS OF THE EXPERIMENTAL CASE}

Usually the actual operation of the network storage requires larger professional operating environment, which is not available in the actual teaching context. This course needs an environment that is easy to access, and meets the network storage functional and performance requirements. Compared to the generality of network storage, our experimental environment is on the Intel Atom embedded platform based on general x86 architecture. We used Intel Atom development board DE2i-150 as our platform that combines an Intel embedded N2600 processor with a high performance processing and configurability. It has 128MB SDRAM, 4MB SSRAM and 64MB flash. This environment will benefit from the compact size, low power consumption and high performance. So it is a suitable platform to deploy the practical contents, including embedded Linux and iSCSI etc. [1, 14-16, 21]. The environment can fully enhance students' cognitive and practical ability for the storage system, the teamwork, and the problem-solving abilities through experiments [27]. iSCSI is an open network storage application. iSCSI technology win the hearts of many CIOs(Chief Information Officer) and storage administrator with its low construction cost and good storage performance. It has been gradually into the enterprise applications, this technology promote the enterprise transformed to centralized storage environment.

\subsection{SYSTEM STRUCTURE}

The case implements small-scale iSCSI network storage system based on Intel Atom embedded platform and Debian [28]. The main hardware contains three parts (as shown in Figure 1):

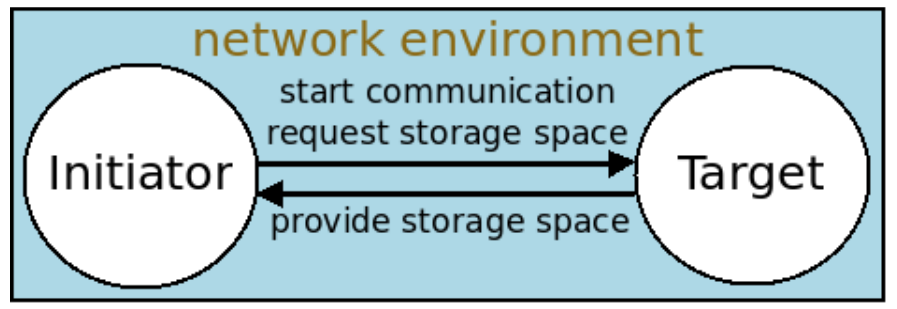

Figure 1. Hardware Environment

Here initiator starts communication by requesting storage space from target. Target then provides enough storage space. Both initiator and target are Intel Atom embedded platforms deployed into Developing Device Pool of the remote laboratory [21]. Network device connects initiator and target by providing network runtime environment for 
iSCSI.

\subsection{PRACTICAL CONTENTS}

The experiment case contains two sections: iSCSI fundamental and advanced experiments. It will cultivate students' practice and teamwork ability via optimizing the experiment environment.

\subsection{1. iSCSI Fundamental Experiment}

Herein, in order to get the experience about iSCSI and embedded Linux through use of Intel Atom, students need to finish the following tasks: building iSCSI environment, configuring iSCSI as well as system testing.

(1) Building iSCSI environment. In this step, students are required to build a basic hardware environment, which contains three Atom boards and a network device. Students need to connect them and make the whole system workable (Figure 2). Linux operating system is needed to be installed on Atom board. In this case, Debian, which is customized for this board, has been installed.

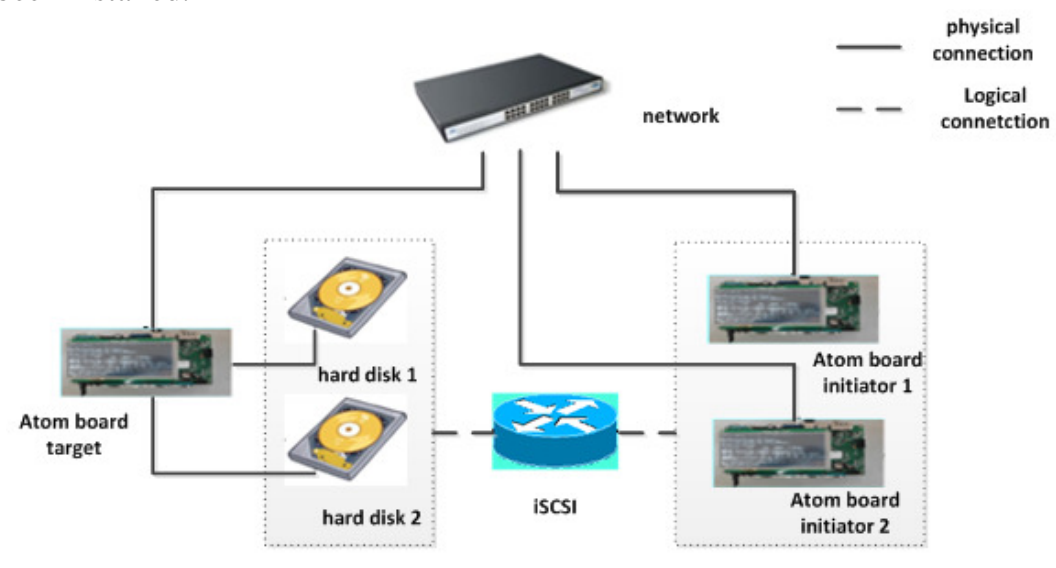

Figure 2. iSCSI Fundamental Experimental Environment.

(2) Configuring. As the case is configured, Atom boards are configured as initiator and target. In this case, two initiators and one target would be needed so that target could map its storage space to initiators through iSCSI and initiators could regard this space as its own space.

a. Experiment of initiators configuration: installing open-iscsi tool, modifying configuration and rebooting initiators' iSCSI service, connecting and verifying state of target, logining target and examining map information, using the space mapped.

b. Experiment of target configuration: loading extra Linux module iscsi_trgt, creating storage space to be mapped, then modifying configuration and rebooting initiators' iSCSI service.

(3) System Testing. After the environment is configured, the tests on performance and function will be required (See Experiment performance test).

\subsubsection{ISCSI ADVANCED EXPERIMENT}

The iSCSI practice experiment is a specific application based on fundamental experiment, including mapping disk and controlling through browser. 


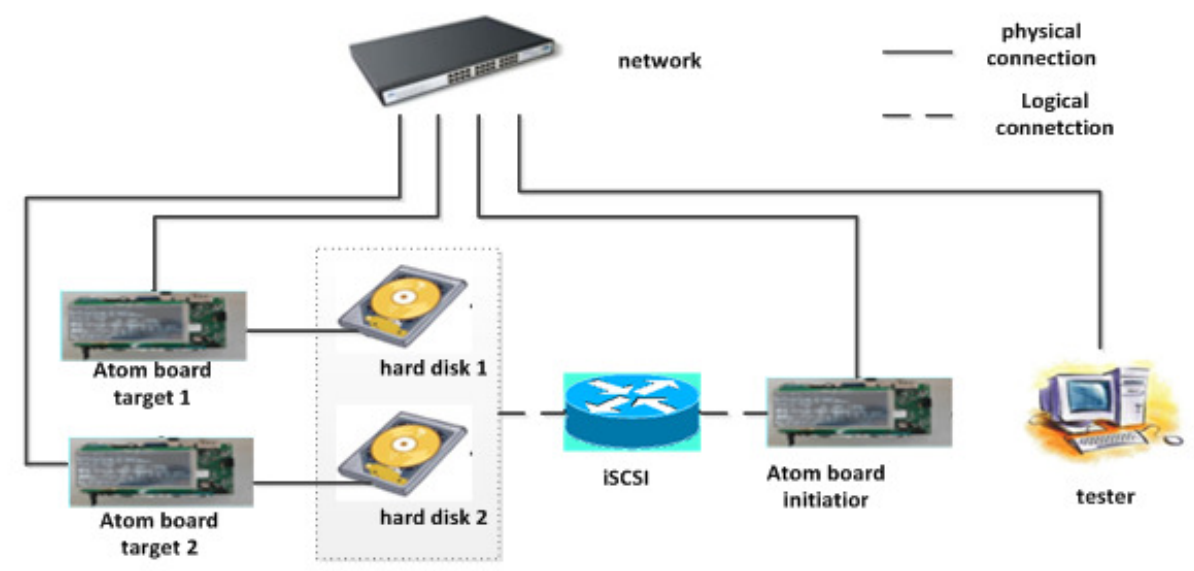

Figure 3. iSCSI Advanced Experimental Environment.

(1) Configuration. The difference with the fundamental experiment is that there are two targets and one initiator in this case. Target system maps its local disk space to initiator through iSCSI, and initiator operate this mapping space in local disk. Once configured, students can use the test client to implement the iSCSI applications (Figure 3). In this step, the target and initiator configuration is the same to the fundamental experiment. Then target 1 and target 2 maps the initiator of disk 1 and disk 2 separately (see Figure 4).

In Figure 4, sdb1 and sdc1 are the results showing two disks mapped and formatted by initiator. They can be operated by initiator as normal disks. Also initiator can add Apache service to support test applications.

(2) Testing Application. Two disks mapped by target are mounted on /var/www/test and /var/www/test1 directories. Then students need to test the process after copying the picture (002ab.jpg) to test directory and copying the track (daoxiang.mp3) to test1 directory (Figure 5). After these steps, the picture and music can be accessed through browser in tester because that Apache server can provide Web access.

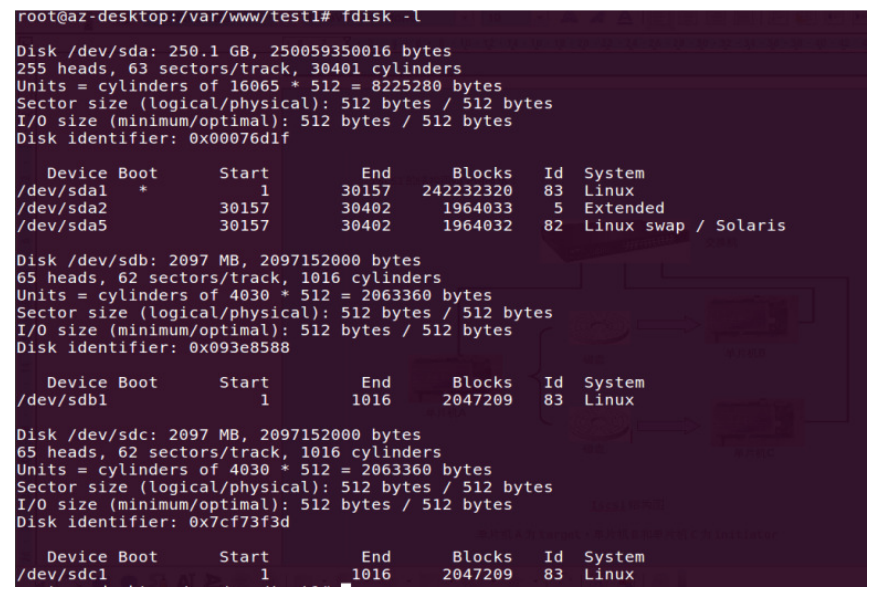

Figure 4. State of Mapped Disks. 


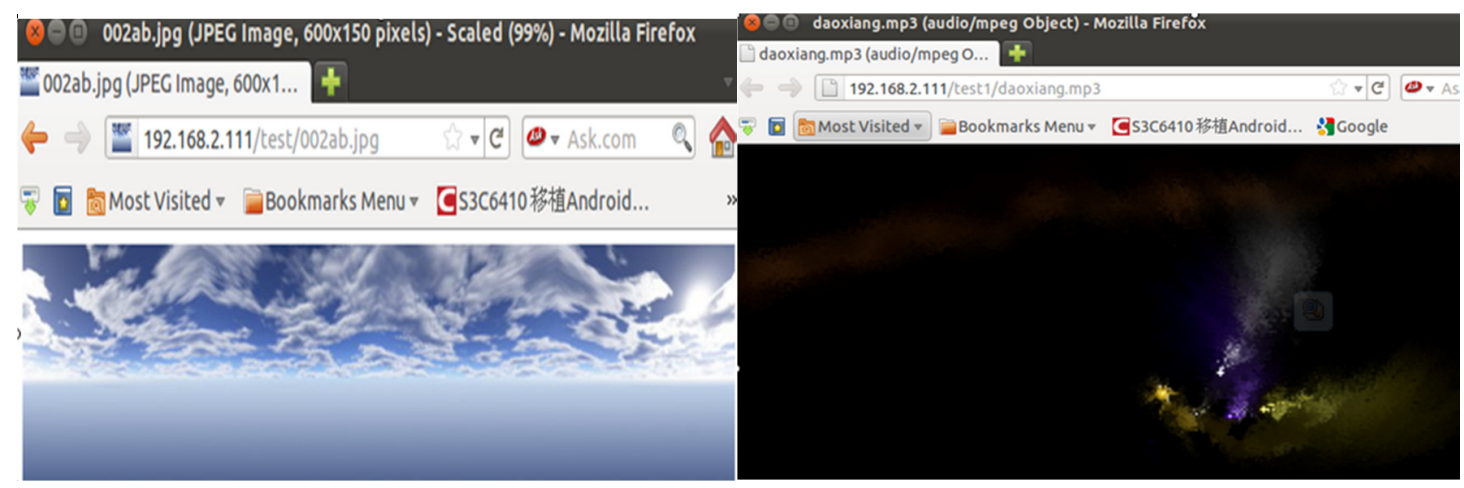

Figure 5. Testing Application

\subsection{EXPERIMENT PERFORMANCE TEST BY STUDENTS}

In this design, students are testing the input and output of the initiator and target by using the Bonnie++ tools [32], which are popular in Linux 22. The specific test environment is shown in Table 1 [34].

Table 1: System Benchmark Environment

\begin{tabular}{ll}
\hline Initiator and target & Atom experimental platform \\
\hline Operating system & Linux (kernel 2.6.26), Debian (lenny) \\
Disk & SATA 2.0/7200r \\
Switch & Brocade 300 \\
Test Software & Bonnie++ $1.03 \mathrm{e}$ \\
The size of the test file & $1000 \mathrm{MB}$ \\
Test memory & $256 \mathrm{MB}$ \\
Network connection speed & $150 \mathrm{Mbps}$ \\
\hline
\end{tabular}

In this case, benchmark needs be done for ten times by using Bonnie ++ , and Table 2 [34] shows the average performance metrics after ten tests. The test result shows that the character based read/write performance between local disk and mapped disk is not very different, but for data block, write/read performance of local disk is much faster than the mapped disk. It is obvious that the performance bottleneck is the communication network in this system. Overall, Atom experimental platform has been able to meet the requirements of network storage throughput and $\mathrm{CPU}$ utilization in this case.

Table 2: Results of System Benchmark

\begin{tabular}{llll}
\hline DISK & & Target disk & Initiator disk \\
\hline \multirow{3}{*}{ Serial Input } & Character(k/sec) & 96 & 92 \\
& CPU Utilization & 98 & 96 \\
& Block(k/sec) & 20587 & 10146 \\
& CPU share & 12 & 9 \\
Serial & Character(k/sec) & 561 & 492 \\
Output & CPU Utilization & 99 & 97 \\
& Block(k/sec) & 80522 & 19897 \\
& CPU share & 12 & 5 \\
\hline
\end{tabular}

\section{EVALUATION OF THE CASE}

\subsection{QUESTIONNAIRE ABOUT THE EXPERIMENTAL CASE}

The students were asked to give feedback at the end of the semester. The questionnaire evaluates the experimental case from the angle of case practicality, case difficulty level and relevance of teaching. The answer of each question was divided into five satisfaction level that is 1 to 5 . The 1 represent the lowest level and 5 represent the highest. After the questionnaire survey, we classified all of the questions to 7 classes, each class corresponding to the item of Table 3. We got the data of table 3 after classifying and statistics. From Table 3, we can know 93.3\% students agree this case teaching could improve the ability of their hands-on practices and $90 \%$ this case teaching can improve their understanding on the storage technology. From the questionnaire result, the cast teaching has effect on students' study 
for 'Network Storage and Security' and gets the students recognition.

Table 3: Feedback on the students' Attitude

\begin{tabular}{|c|c|c|c|c|c|}
\hline$\frac{\text { Satisfaction level }}{\text { Criteria }}$ & 1 & 2 & 3 & 4 & 5 \\
\hline $\begin{array}{l}\text { Case teaching removes the monotony of purely theoretical } \\
\text { knowledge. }\end{array}$ & 0 & 0 & $3.3 \%$ & $40 \%$ & $56.7 \%$ \\
\hline $\begin{array}{c}\text { Case teaching could improve the ability of our hands-on } \\
\text { practices. }\end{array}$ & 0 & 0 & 0 & $6.7 \%$ & $93.3 \%$ \\
\hline $\begin{array}{l}\text { Case teaching promotes the exchanges between teachers and } \\
\text { students' peer-based learning. }\end{array}$ & 0 & 0 & $6.7 \%$ & $40.0 \%$ & $53.3 \%$ \\
\hline Case teaching effectively reduces errors in the experiment. & 0 & 0 & $16.7 \%$ & $43.3 \%$ & $40 \%$ \\
\hline $\begin{array}{l}\text { Case teaching can improve my understanding on the storage } \\
\text { technology efficiently. }\end{array}$ & 0 & 0 & 0 & $10 \%$ & $90 \%$ \\
\hline $\begin{array}{c}\text { Case teaching can be popularized to other computer basics } \\
\text { courses. }\end{array}$ & 0 & $16.7 \%$ & $26.7 \%$ & $33.3 \%$ & $23.3 \%$ \\
\hline I like case teaching. & 0 & $10 \%$ & $10 \%$ & $17 \%$ & $63 \%$ \\
\hline
\end{tabular}

\subsection{EFFECT COMPARISON BETWEEN CASE TEACHING CLASS AND TRADITIONAL TEACHING} CLASS

After the questionnaire survey, we have the evaluation of the improvements with the implementation of our experimental case was conducted in two classes with 30 students each. The students score was composed of formative tests, experimental results and the final exam. Formative testing was a means of systematic evaluation, which was in accordance with the teaching objectives in the overall teaching process. The classes are marked as $\mathrm{Ga}$ and $\mathrm{Gb}$, where $\mathrm{Ga}$ used the case reported in this paper and $\mathrm{Gb}$ is the traditional teaching class. Table 4 below is the result of each class, and Figure 6 is the histogram about it. From these, we can see the number of high score ( $>=90$ and $>=80)$ in case teaching class more than the traditional teaching class. And there is no student's score below 60 in case teaching class. These achievements show that our case can improve their theoretical and manipulative ability and reach the expected effect. It is implied that learning outcome has been improved.

Table 4: Testing result of each class

\begin{tabular}{|c|c|c|}
\hline Score Number & $\begin{array}{l}\text { Case teaching } \\
(G a)\end{array}$ & $\begin{array}{c}\text { Traditional teaching } \\
(G b)\end{array}$ \\
\hline$>=90$ & 3 & 1 \\
\hline$>=80$ & 8 & 3 \\
\hline$>=70$ & 11 & 14 \\
\hline$>=60$ & 8 & 10 \\
\hline$<60$ & 0 & 2 \\
\hline
\end{tabular}




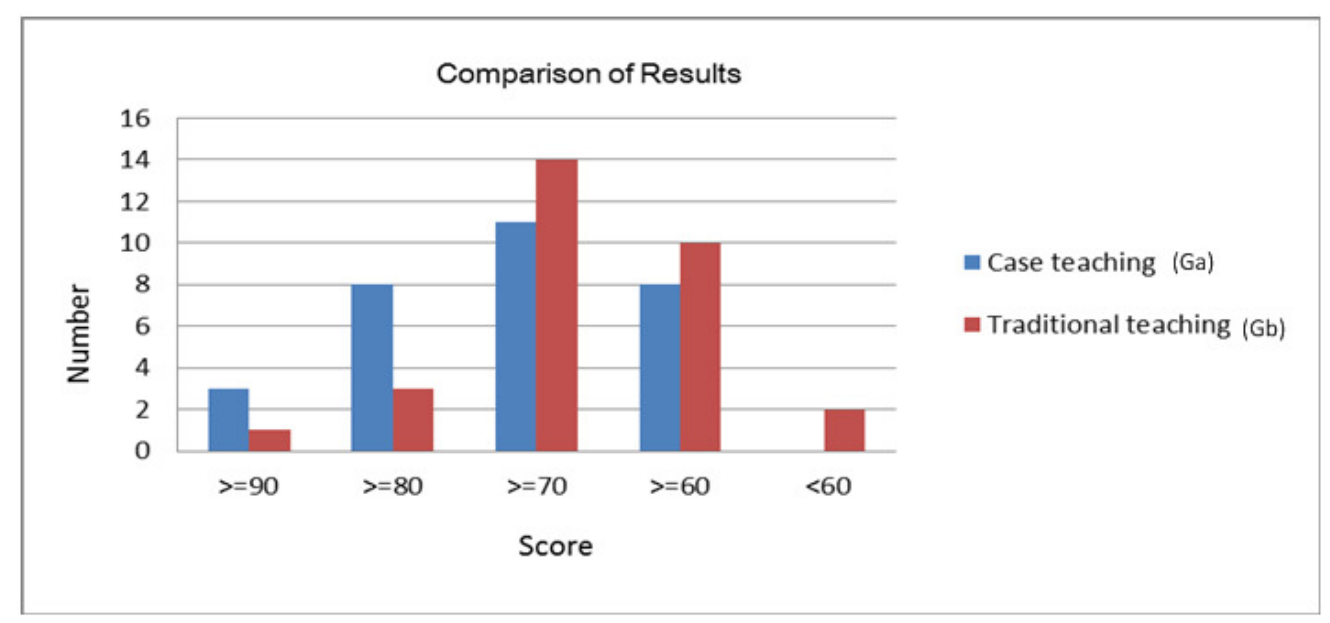

Figure 6. Comparison of Results

Furthermore, we randomly extracted one examination paper from the 80 to 100 section of $\mathrm{Ga}$ and $\mathrm{Gb}$ respectively (named A and B), we compared and analyzed the answers of same test question. We found that the answer of $\mathrm{A}$ explained this question from the two aspects of theory and practice and got a higher score. However, the answer of B just from the angle of theory to answer this question and it is worse than the theory part of $\mathrm{A}$. This result shows that the student A had a better understanding of theory than B, it reflect directly the experiment case can enhance the students' understanding of theoretical knowledge, at the same time, this advantage can be reflected in the examination and it will enable students to get a better score.

\subsection{COMPREHENSIVE EVALUATION OF STUDENTS ABOUT THE EXPERIMENTAL CASE}

For this part, we ask 30 students in Ga class answer the question "How to evaluate this experimental case", which include for option, very satisfied, satisfied, qualified, and unsatisfied. As we seen in Figure 7 [34], $90 \%$ of students in the experimental teaching are generally positive.

According to the above feedback, we also found the further design of new experiment content also would also suffer from a limitation for scaling up, mainly due to the inadequacies of the limited number of existing hardware devices and the longer time for organizing experiments.

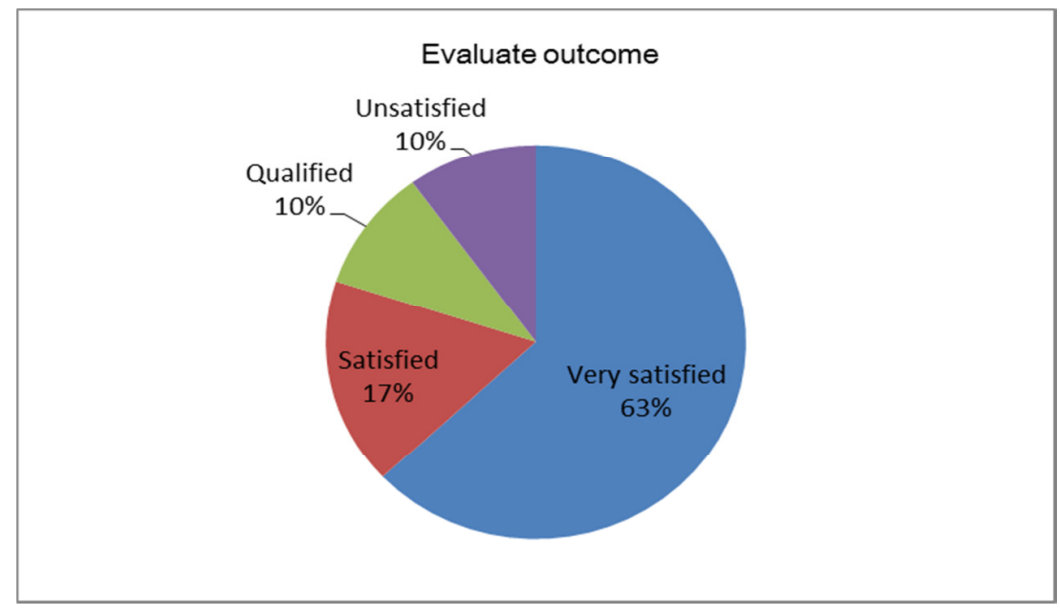

Figure 7. Students' Evaluation of the Case

\section{CONCLUSION AND FUTURE WORK}

This paper shows that using experimental case can improve the students' practical ability in the courses on network storage, and it can effectively help to improve students' enthusiasm for learning and consolidate their learning outcomes. We have completed statistical analysis and given the evaluation on the experimental teaching. $90 \%$ of the feedback is positive. 
Based on this teaching method, the students can conveniently arrange their own learning and working progress in the environment, conduct experiments on their own creative ideas with regard to embedded systems, and interact with each other for some common goals. Especially, all of these users are now able to gain hands-on experiences of embedded devices according to their own schedule, and progress on their individual basis with the minimum conflicts in the occupation of available devices. It is easy to get started and has a good versatility, and it can also stimulate students' design ability and innovation desire, which not only expand the scope of the students' knowledge, but also open up new directions for their employment options. After the students' answer in exam paper analysis, teaching strategy will impact the test score directly, the students in our course have a higher test score.

The Intel's university research program is a long term strategic program. The objective is to initialize and develop the research collaboration with universities in China, thus to develop and progress knowledge learning together. Intel supported hardware and software, like the Intel Atom processor platform as mentioned above. As a successful example, the achievements and outcome reported in this paper could apply to the corresponding courses. Other universities can use the experimental case to greatly enhance students' practical ability and effectively improve the course learning outcome.

In future we will explore this capacity to further increase and improve the experiment content, to help the students gain more storage expertise and technology, expand professional knowledge, enhances practical ability. We may also reduce the complexity of the experiment. We will involve a detailed evaluation on the designed solution in the classroom, where we will include an observational study of the participants' collaborative interactions, testing data and learning outcomes.

\section{ACKNOWLEDGMENTS}

This work was supported by National Natural Science Foundation of China under Grant No. 61402210 and 60973137, Program for New Century Excellent Talents in University under Grant No. NCET-12-0250, Gansu Sci.\&Tech. Program under Grant No. 1104GKCA049, 1204GKCA061 and 1304GKCA018. The Fundamental Research Funds for the Central Universities under Grant No. lzujbky-2014-49, lzujbky-2013-k05, lzujbky-2013-43, lzujbky-2013-44 and lzujbky-2012-44, Gansu Telecom Cuiying Research Fund under Grant No. 1zudxcy-2013-4, Google Research Awards and Google Faculty Award, China.

\section{REFERENCES}

[1] Mehran Sahami,Mark Guzdial,Andrew McGETTRICK,Steve Roach:Setting the stage for computing curricula 2013: computer science -report from the ACM/IEEE-CS joint task force:SIGCSE '11 Proceedings of the 42nd ACM technical symposium on Computer science education,2011,161-162.

[2] Cagiltay, N. E. (2007). Teaching software engineering by means of computer-game development: challenges and opportunities. British Journal of Educational Technology, 38(3), 405-415.

[3] Egenfeldt-Nielsen, S. (2007). Third generation educational use of computer games. Journal of Educational Multimedia and Hypermedia, 16(3), 263-281.

[4] Chuo-Bin Lina,Shelley Shwu-Ching Youngb,Tak-Wai Chana,Yen-Hua Chena(2005) Teacher-oriented adaptive Web-based environment for supporting practical teaching models: a case study of "school for all" Computers \& Education 44(2) 155-172.

[5] Cuneyt Bayilmis ,Development of a web_based educational interface using matlab builder ne with web figure for digital modulation techniques, 2010 .Computer Applications in Engineering Education,20(4) 604-610.

[6] Seiji Isotania,Riichiro Mizoguchic, Sadao Isotanib , A semantic webbased authoring tool to facilitate the planning of collaborative scenarios compliant with learning theories,2013,Computers \& Education 63,2012,267-284 1

[7] Yee Leng Eowa,Wan Zah bte Wan Alib,Rosnaini bt. Mahmudb,Roselan Bakic (2010) Computer games development and appreciative learning approach in enhancing students' creative perception Computers \& Education 54(1),146-161.

[8] Yueh-Min-Huang,Yen Ting Lin,Shu-Chen Cheng:Effectiveness of a Mobile Plant Learning System in a science curriculum in Taiwanese elementary education:Computers \& Education,2010, 47-58.

[9] Farily,M.Building Storage Networks:McGraw Hill 2011.

[10] Preton,C.Using SAN and NAS:O'Reilly 2002.

[11] Vladan Jovanovic,Timur Mirzoev:Teaching Network Storge Technology - Assessment Outcomes and Directions:SIGITE’08, 2008, Cincinnati, Ohio, USA.

[12] Charlie Kaufman, Radia Perlman, Mike Speciner, Network Security, Private Communication,2002.

[13] Erik Riedel, Mahesh Kallahalla, and Ram Swaminathan, A framework for evaluating storage system security Proc. First USENIX Conference on File and Storage Technologies (FAST)., 15-30.

[14] Kalman Z. Meth, Julian Satran, Design of the iSCSI Protocol, Mass Storage Systems and Technologies, 2003.(MSST 2003). Proceedings. 20th IEEE/11 th NASA Goddard Conference on. IEEE,116-122.

[15] Dan Zhou, Li Ou, Xubin (Ben) He, Stephen L. Scott: Online Remote Data Backup for iSCSI-based Storage.Systems: Proceedings of the Internet Computing: 2004.

[16] Meth, K.Z.Design of the iSCSI protocol Mass Storage Systems and Technologies, 2003. (MSST 2003). Proceedings. 20th IEEE/11th NASA Goddard Conference, 116-122. 
[17] Zhangdong. The Westward storage - network storage systems principles refined solution with best practice Beijing: Tsinghua University Press, 2008.

[18] Xu Min ; Sch. of Inf., REMIN Univ. of China, Beijing, China ; Zhou Lijuan:Teaching Experiences on Course of Comprehensive Practice of Program Design:Information Technology and Applications, 2009. IFITA'09. International Forum on. IEEE, 3: 573-575.

[19] Han-Yu Sunga,Gwo-Jen Hwang A collaborative game-based learning approach to improving students' learning performance in science courses Computers \& Education ,2012,63, 43-51.

[20] Hae Won Park, Howard: A.: Providing Tablets as Collaborative-Task Workspace for Human-Robot Interaction: 2013 8th ACM/IEEE International Conference, Page(s): 207-208, 2013.

[21] Zhou Rui,Zhou Qingguo,Cheng Guanghui,Wang Bbaojun,Li Lian and Nicholas McGuire:An Open Source and Network-based Remote Laboratory for Embedded Systems: International Journal of Engineering Education,2010.

[22] Jianhua Zhao, Xiaohua Li: Comprehensive Evaluation on the Practice Ability of Undergraduate: 2008 International Symposium on Knowledge Acquisition and Modeling, Page(s):434 - 437.

[23] Zhao Yan jun, Li Min jie, Zhang Chun ying, Wang Jing: Innovative Practices Teaching Mode Research of the Fundamentals of Computer : Computer Science \& Education (ICCSE), 2013 8th International Conference, Page(s): 1154-1159, 2013.

[24] Verner, J.M., Sampson, J., Tosic, V., Bakar, N.A.A., Kitchenham, B.A.: Guidelines for Industrially-Based Multiple Case Studies in Software Engineering: Research Challenges in Information Science, 2009. RCIS 2009. Page(s): 313-324.

[25] Watts, J. , Huiming Yu, Xiaohong Yuan: Case study: Using Smart Cards with PKI to implement data access control for health information systems: IEEE SoutheastCon 2010 (SoutheastCon), Page(s): 163-167, 2010.

[26] de la Torre, L., Heradio, R., Jara, C., Sanchez, J., Dormido, S., Torres, F., Candelas, F.: Providing Collaborative Support to Virtual and Remote Laboratories, Learning Technologies, IEEE Transactions on (Volume:PP, Issue: 99 ), 2013.

[27] Kwangsun Ko, Gu Su Kim, June Kim, JungHyun Han, Ungmo Kim, Young Ik Eom: Design and Implementation of a Security Framework Based on the Object-Based Storage Device Standard: Computational Science and Its Applications - ICCSA 2006,1028-1035.

[28] Beavers, B., The Story behind the Intel Atom Processor Success:Design \& Test of Computers, IEEE,March-April 2009 ,26(2),8-13.

[29] Bonnie++. http://www.coker.com.au/bonnie++/. 2012-01-20.

[30] Chun-Chia Wang, Hsuan Pu Chang, Shu-Wei Yeh, Shih, T.K, Mei-Chung Chen: Supporting collaborative learning environment with learning blogs: Ubi-Media Computing, 2008 First IEEE International Conference, Page(s): 553-557, 2008.

[31] Javier García , Joaquín Entrialgo. Implement a Low Cost Laboratory for the Teaching of Storage Area Networks. Comput Appl Eng Educ 9999: 1-9, 2015

[32] LIANG Zheng-ping, WANG Zhi-qiang, SHEN Xiang-jun, CHEN Jian-yong, Exploration and Practice of Curriculum Teaching on "Networked Storage Technology", Computer Education, 2009, No.87(03) 98-101.

[33] HUANG Li-qin, Course Construction and Teaching Practice of Network Storage Technology, Journal of Jiangsu Vocational and Technical Institute of Economics and Commerce, 2013, (5).

[34] Zhou Rui, Ai Zhu, Di Chang, Li Jun, Lv Qingquan, Zhou Qingguo, The Design and Implementation of Network Storage Teaching Case Based on Atom Platform, Computer Education, 2012,(21). 